\title{
Editorial to Special Issue on Smart Environments
}

\author{
Diedrich Wolter ${ }^{1} \cdot$ Alexandra Kirsch $^{2}$
}

(c) Springer-Verlag GmbH Deutschland 2017

Smart environments are sometimes regarded as ubiquitous systems comprising sensors, actuators, interaction components and computational elements connected as a network-but this picture is misleading just like picturing a smartphone merely as a mobile computing device would ignore its vital role within modern society for communication, organisation of business and daily life, and entertainment. The area of smart environments should thus be seen as installations supporting and enhancing the abilities of humans in their regular life as well as improving the environments themselves, for example in terms of energy efficiency. Smart environments are based on the technical substrate sketched above but they bear more challenges than the seamless composition of its components addressed in current research in technical disciplines. Setting technical challenges aside, developing intuitive interfaces to a system hidden in the environment and enabling the system to provide suitable assistance for a wide range of every-day tasks necessitates answers to manifold research questions. Indeed, at present smart environments are best pictured as an active field of research spanning several disciplines. Smart environments spans across a whole variety of core AI questions, but already a limited degree of 'smartness' can present a useful enhancement in some application contexts. This renders smart environments not only an

Diedrich Wolter

diedrich.wolter@uni-bamberg.de

Alexandra Kirsch

alexandra.kirsch@uni-tuebingen.de

1 Faculty of Information Systems and Applied Computer Sciences, University of Bamberg, Bamberg, Germany

2 Department of Computer Science, Eberhard Karls Universität Tübingen, Tübingen, Germany interesting application area for contemporary AI research, but the challenges faced here are important stages towards long-term AI goals. Moreover, a careful design of interaction is necessary when developing smart environments to make their benefits intuitive and accessible to users. Designing effective modes of interaction has challenges in its own right, exceeding modalities and paradigms of classical approaches to human-machine interaction by far. For example, natural interaction with smart environments needs to be situated, that is, aware of and responsive to environmental and cognitive states. The ubiquitous nature of smart environments calls for implicit means of interaction. Moreover, systems capable of autonomous actions may shift initiative in interaction.

All these research questions sketched above are further motivated by important applications which particularly concern enabling independent living, comfort, and safety. Since all applications penetrate our daily life with sensors, privacy becomes a central issue too.

With this special issue we aim to portray the manifold AI-related research currently being performed in this area. In particular, this special issue aims to shed a light on the contact areas and the interplay of AI and human-machine interaction. Since much research in this area is also technology-driven, we have chosen to pay tribute to technology as an enabling factor by inviting "lab reports". These reports are similar to project reports that portray individual research activities, but they focus on the technological substrate underlying research activities. Indeed, research activities are at the hearth of this special issue. We characterise the area of smart environments by the research questions it triggers and articles in this issue shed a light on how some of these question can be answered. 


\section{Content}

1.1 Survey

- Smart Environments: What is it and why should we care? Diedrich Wolter and Alexandra Kirsch

\subsection{Technical Contributions}

- Indoor Localisation with Beacons for a User-Friendly Mobile Tour Guide

Tobias Kaulich, Thomas Heine, Alexandra Kirsch

- Towards Identifying User-Centered Requirements for Smart In-House Mobility Services

Dorothee Rocznik, Klaus Goffart, Manuel Wiesche, and Helmut Krcmar

- Situated Interaction with a Smart Environment: Challenges and Opportunities

Thora Tenbrink

\subsection{Research Projects}

- Living Lab Bamberg: An Infrastructure to Explore Smart City Research Challenges in the Wild

Aboubakr El Hacen Benabbas, Golnaz Elmamooz, Brent Lagesse, Daniela Nicklas, and Ute Schmidt

- "KogniChef": A Cognitive Cooking Assistant Alexander Neumann, Christof Elbrechter, Nadine PfeifferLeßmann, Risto Köiva, Birte Carlmeyer, Stefan Rüther, Michael Schade, André Ückermann, Sven Wachsmuth, and Helge J. Ritter

- Plug and play your Robot into your Smart Home Sao Mai Nguyen, Christophe Lohr, Philippe Tanguy, and Yiqiao Chen

- The Spatial Interaction Laboratory Jasper van de Ven and Frank Dylla

- The Cognitive Service Robotics Apartment Sebastian Wrede, Christian Leichsenring, Patrick Holthaus, Thomas Hermann, and Sven Wachsmuth

\subsection{Interview}

- Interview with Prof. Dr. Daniel Buhr, Professor for Policy Analysis and Political Economy at the University of Tübingen

Alexandra Kirsch

\section{Service}

2.1 Conferences and Workshops

- International Joint Conference on Artificial Intelligence (IJCAI)

- AAAI Conference on Artificial Intelligence (AAAI)

- European Conference on Artificial Intelligence (ECAI)

- German Conference on Artificial Intelligence (KI)

- ACM International Joint Conference on Pervasive and Ubiquitous Computing (UbiComp)

- International Conference on Intelligent Environments (IE)

- International Conference on Ambient Intelligence (ISAmI)

- International Conference on Ubiquitous Computing and Ambient Intelligence (UCAmI)

- International Conference on Autonomous Agents and Multiagent Systems (AAMAS)

- International Conference on Human-Robot Interaction (HRI)

- IEEE International Symposium on Robot and Human Interactive Communication (RO-MAN)

- Conference on Human Factors in Computing Systems (CHI)

- AAL Forum

\subsection{Journals}

- Journal of Ambient Intelligence and Smart Environments http://www.iospress.nl/journal/journal-of-ambient-intelligence-and-smart-environments/

- Journal of Ambient Intelligence and Humanized Computing http://www.springer.com/journal/12652

- Proceedings of the ACM on Interactive, Mobile, Wearable and Ubiquitous Technologies (IMWUT) http:// imwut.acm.org/

- ACM Transactions on Computer-Human Interaction https://tochi.acm.org/

- IEEE Transactions on Systems, Man, and Cybernetics, Part C (Applications and Reviews) http://ieeexplore. ieee.org/servlet/opac?punumber $=5326$

- International Journal of Social Robotics https://link. springer.com/journal/12369 


\subsection{Books}

- Smart Environments: Technology, Protocols and Applications

Diane J. Cook and Sajal K. Das (editors), Wiley, 2004

- Intelligent Environments: Methods, Algorithms and Applications

Dorothy Monekosso, Yoshinori Kuno, Paolo Remagnino (editors), Springer, 2009
- Handbook of Ambient Intelligence and Smart Environments

Hideyuki Nakashima, Hamid Aghajan, and Juan Carlos Augusto (editors), Springer, 2010 